\title{
Posterior Septal Widening as a Cause of Nasal Airway Obstruction
}

\author{
Mofiyinfolu Sokoya*, Henry Barham
}

Department of Otolaryngology—Head and Neck Surgery, University of Colorado, Aurora, CO, USA

Email: ^fiyin.sokoya@ucdenver.edu

How to cite this paper: Sokoya, M. and Barham, H. (2016) Posterior Septal Widening as a Cause of Nasal Airway Obstruction. Open Access Library Journal, 3: e3263.

http://dx.doi.org/10.4236/oalib.1103263

Received: November 23, 2016

Accepted: December 11, 2016

Published: December 14, 2016

Copyright $\odot 2016$ by authors and Open Access Library Inc.

This work is licensed under the Creative Commons Attribution International License (CC BY 4.0).

http://creativecommons.org/licenses/by/4.0/

(c) (i) Open Access

\begin{abstract}
Nasal airway obstruction, a well-known cause of patient discomfort, is one of the most common presenting complaints to the otolaryngologist. The different causes of nasal airway obstruction are very wide-ranging, spanning from congenital life threatening causes to acquired benign causes. Herein, we present a case of nasal obstruction caused by widening of the posterior septum. A 52-year-old gentleman presented to the rhinology clinic with complaint of chronic nasal congestion for 4 years. Trials of topical steroid sprays and nasal saline rinses had been unsuccessful in symptomatic resolution. On anterior rhinoscopy, the septum was noted to be straight anteriorly, but appeared to be deviated bilaterally, flaring into the nasopharynx and obstructing the airway. A CT scan was performed, which showed soft tissue enlargement on the posterior aspect of the septum. The patient was subsequently taken to the operating room for a posterior septectomy and inferior turbinectomy. He reported alleviation of symptoms postoperatively. It is important for the otolaryngologist to be aware of the myriad of causes of nasal airway obstruction. An accurate history and physical examination including anterior and posterior rhinoscopy is important in the diagnosis of nasal airway obstruction. More importantly, the physician should be aware of the possibility of a widened posterior septum as a cause of nasal airway obstruction.
\end{abstract}

\section{Subject Areas}

Otorhinolaryngology

Keywords

Nasal Septum, Choanal Stenosis, Endoscopy, Septoplasty

\section{Introduction}

Nasal airway obstruction is one of the most common presenting complaints in otola- 
ryngology. Different causes of nasal airway obstruction range from congenital life threatening causes to acquired benign causes. Choanal atresia is an anatomic narrowing of the posterior choanal passageway. This narrowing can exist in a spectrum of mild stenosis to complete atresia. The first case of choanal atresia was described by Roederer in 1755 [1]. Over the past 250 years, our understanding of the anatomy and embryology involved in the formation of this congenital anomaly has improved. Reports suggest that $90 \%$ of choanal obstruction is of a skeletal abnormality, portending a bony obstruction, while $10 \%$ is of a membranous etiology [2]. Multiple embryological models have been postulated to explain the development of choanal stenosis and atresia. These include failure of obliteration of the buccopharyngeal membrane from the foregut, persistence of the nasobuccal membrane of Hochstetter, presence of mesodermal adhesions in the choanae, and finally a misdirection of mesodermal development secondary to local genetic factors [3]. The migration of neural crest cells from the dorsal neural folds around the $4^{\text {th }}$ week of gestation begins the development of nasal architecture. This is completed by the $12^{\text {th }}$ week of gestation. This process is affected by the presence of various genetic and molecular factors including hyaluronic acid and retinoic acid. If the migration of these neural crest cells is altered, the formation of the structures in the primitive nose, including the nasal pits and choanae is hindered. Herein, we present a case of nasal airway obstruction caused by widening of the posterior septum, a mild but clinically significant form of membranous choanal stenosis. This case highlights the importance of nasal endoscopy in the diagnosis of nasal airway obstruction. A recent survey of otolaryngologists reports that nasal endoscopy was considered a criterion standard in the evaluation of nasal airway obstruction [4], corroborating the central theme of this case report.

\section{Case Presentation}

A 52-year-old gentleman presented to the rhinology clinic with complaint of chronic nasal congestion for 4 years. He had undergone evaluation and treatment by multiple primary care doctors and otolaryngologists with trials of topical steroid sprays and nasal saline rinses, with no success. On anterior rhinoscopy the septum appeared straight anteriorly, but posteriorly appeared to deviate bilaterally, flaring into the nasopharynx and obstructing the airway. A CT scan was performed, which showed soft tissue enlargement on the posterior aspect of the septum (Figure 1). After discussion of the surgical risks and benefits, he was subsequently taken to the operating room for an endoscopic posterior septectomy and inferior turbinectomy. He reported alleviation of symptoms postoperatively.

\section{Discussion}

Choanal atresia may present as bilateral, unilateral or as a syndromic feature. Bilateral choanal atresia presents at birth as asphyxia neonatorum. Due to the fact that neonates are obligate nasal breathers, bilateral choanal atresia presents as an acute picture of respiratory distress and cyanosis, which must be quickly identified and treated with securing 


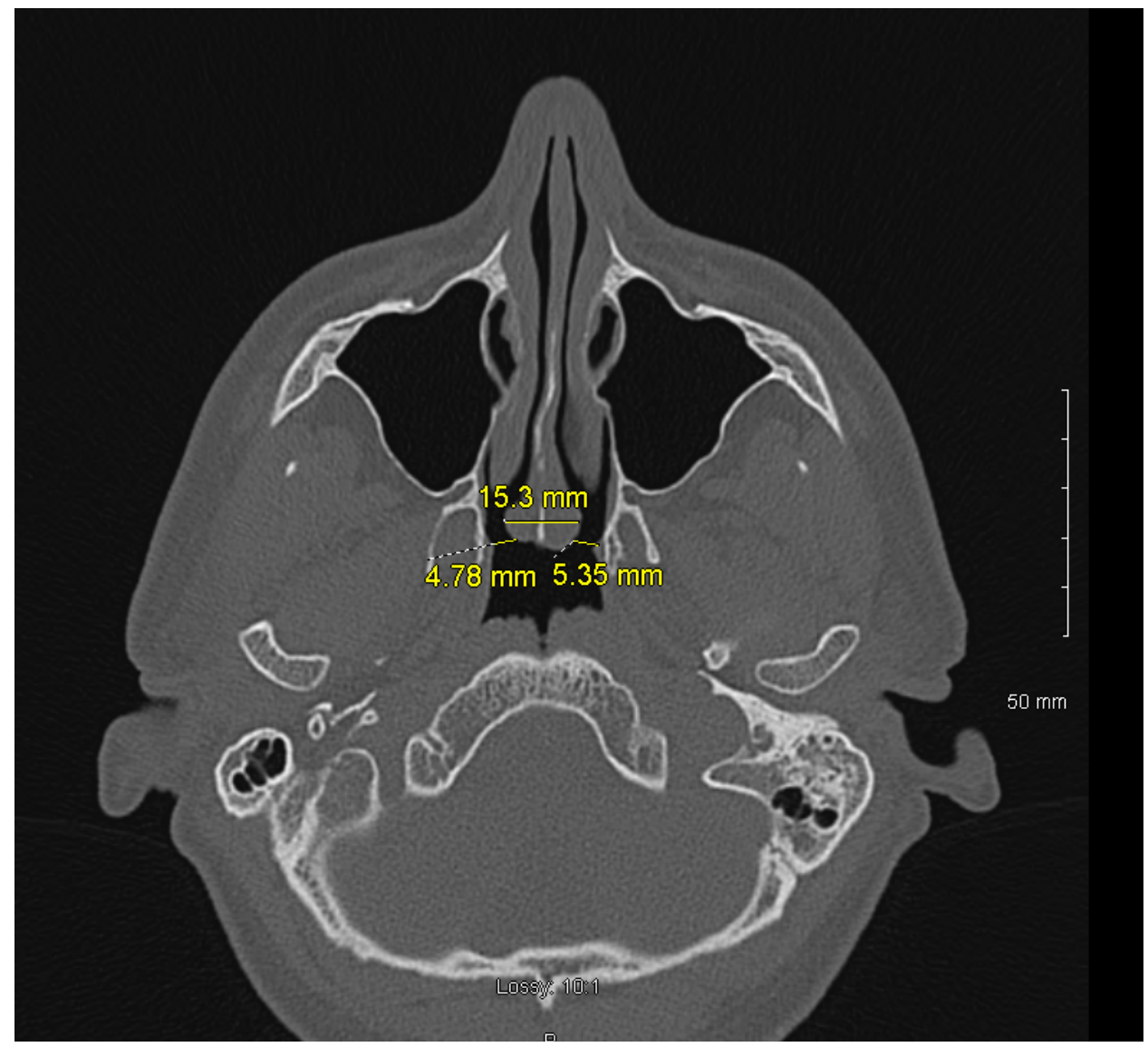

Figure 1. Axial cut computed tomography image showing mucosal widening of the posterior septum causing obstruction of bilateral choanae. There is narrowing of the choanae bilaterally due to mucosal widening of the posterior septum.

the airway. Unilateral choanal atresia rarely causes respiratory distress but often presents as a unilateral nasal obstruction with persistent drainage.

The most common syndrome associated with choanal atresia is the CHARGE association. Literature review shows that close to $30 \%$ of children with choanal atresia had CHARGE syndrome [5], and 59\% of patients with CHARGE syndrome had choanal atresia [3].

Our case presents a milder form of membranous choanal stenosis, deemed posterior septal widening. As seen in Figure 1, there is no widening of the vomer or medialization of the pterygoids, as would be expected in bony choanal stenosis or atresia. Furthermore, the choana is not completely atretic as would be expected in membranous atresia. In this case, we found soft tissue widening of the posterior septum, leading to narrowing of the bilateral choanae. As expected, this deformity was not seen by anterior rhinoscopy, confirming the importance of nasal endoscopy.

A range of surgical techniques has been developed for the treatment of choanal stenosis and atresia. The use of Fearon dilators for transnasal puncture has been widely adopted as a means of establishing an airway. However, the shearing forces involved in this technique cause additional trauma and can lead to eventual restenosis. Hengerer et 
al. reported that combining the transnasal approach with endoscopic resection is associated with lower rates of restenosis [6]. Transpalatal resection has also been shown to be beneficial in the treatment of choanal atresia. In this method, the anterior palatine bone, vomer, and the atretic bone are removed intraorally. Complications of this technique include crossbite deformity, palatal flap breakdown and fistulization [2]. Endoscopic resection has been widely adopted as the superior method of surgical intervention with high success and low revision rates [7] [8].

In otolaryngology, perspectives in the diagnosis of nasal airway obstruction seem to favor the use of nasal endoscopy in the diagnosis of nasal airway obstruction. However $30 \%$ of surveyed otolaryngologists do not consider nasal endoscopy to be a criterion standard in the evaluation of nasal airway obstruction [4]. This difference in opinion potentially leads to missed diagnosis, delay in treatment, and increased patient morbidity.

\section{Conclusion}

Nasal airway obstruction is a common chief complaint in primary care and otolaryngology. Choanal stenosis is a known cause of nasal airway obstruction in infants and adults. Knowledge of the spectrum of choanal stenosis is imperative to otolaryngologists. An accurate history and physical examination including anterior and posterior nasal endoscopy is important in the diagnosis of nasal airway obstruction. More importantly, the physician should be aware of the possibility of a widened posterior septum as a cause of nasal airway obstruction.

\section{References}

[1] Flake, C.G. and Ferguson, C.F. (1964) Congenital Choanal Atresia in Infants and Children. The Annals of Otology, Rhinology, and Laryngology, 73, 458. https://doi.org/10.1177/000348946407300216

[2] Ramsden, J.D., Campisi, P. and Forte, V. (2009) Choanal Atresia and Choanal Stenosis. Otolaryngologic Clinics of North America, 42, 339-352.

https://doi.org/10.1016/j.otc.2009.01.001

[3] Fulmer, R.P., Calhoun, Karen, H. and Quinn, F.B. Choanal Atresia. UTMB Otolaryngology Grand Rounds. http://www.utmb.edu/otoref/grnds/choanal.htm

[4] Shemirani, N.L., Rhee, J.S. and Chiu, A.M. (2008) Nasal Airway Obstruction: Allergy and Otolaryngology Perspectives. Annals of Allergy, Asthma \& Immunology, 101, 593-598. https://doi.org/10.1016/S1081-1206(10)60221-9

[5] Leclerc, J.E. and Fearon, B. (1987) Choanal Atresia and Associated Anomalies. International Journal of Pediatric Otorhinolaryngology, 13, 265-272. https://doi.org/10.1016/0165-5876(87)90107-8

[6] Hengerer, A.S., Brickman, T.M. and Jeyakumar, A. (2008) Choanal Atresia: Embryologic Analysis and Evolution of Treatment, a 30-Year Experience. The Laryngoscope, 118, 862866. https://doi.org/10.1097/MLG.0b013e3181639b91

[7] Stankiewicz, J.A. (1990) The Endoscopic Repair of Choanal Atresia. Otolaryngology-Head and Neck Surgery: Official Journal of American Academy of Otolaryngology-Head and Neck Surgery, 103, 931. 
[8] Deutsch, E., Kaufman, M. and Eilon, A. (1997) Transnasal Endoscopic Management of Choanal Atresia. International Journal of Pediatric Otorhinolaryngology, 40, 19-26. https://doi.org/10.1016/S0165-5876(96)01486-3

Submit or recommend next manuscript to OALib Journal and we will provide best service for you:

- Publication frequency: Monthly

- 9 subject areas of science, technology and medicine

- Fair and rigorous peer-review system

- Fast publication process

- Article promotion in various social networking sites (LinkedIn, Facebook, Twitter, etc.)

- Maximum dissemination of your research work

Submit Your Paper Online: Click Here to Submit

Or Contact service@oalib.com 